\title{
Consórcios e suas barreiras no desenvolvimento de projetos P\&D de tecnologias pré-competitivas: modelo conceitual e aplicação
}

\author{
Reinaldo Lopes Ferreira ${ }^{1 *}$ (1) \\ Cristina Godoy ${ }^{2}$ \\ Eduardo Vasconcellos \\ Fernando Fernandez ${ }^{4}$
}

\section{Resumo}

Este artigo propõe caracterizar as principais barreiras ao desenvolvimento de arranjos colaborativos de pesquisa e desenvolvimento (P\&D) de tecnologias pré-competitivas. As barreiras foram relacionadas a partir da literatura e de depoimentos dos participantes do consórcio de Pesquisa da Tecnologia de Processamento por Atrito e Mistura (C2PA), cujo foco se concentra nas tecnologias de deposição superficial por atrito (friction surfacing - FS) e de soldagem por agitação e fricção (friction stir welding - FSW). Foi avaliado o grau de interferência dessas barreiras sobre esses modelos de cooperação. Os resultados do estudo mostram que as barreiras tendem a refletir cada tipo de formação, por exemplo, se são concorrentes ou não, pelo grau de maturidade das tecnologias desenvolvidas, verificado pela escala TRL (Technology Readiness Level). A pesquisa revelou que as barreiras consideradas mais prejudiciais aos consórcios são aquelas associadas à falta de confiança entre as partes e ao gerenciamento (inadequado) da colaboração. Igualmente, houve ressalvas para as dificuldades decorrentes da cultura de inovação e/ou do mindset de produção da indústria brasileira, que não está habituada a P\&D de longo prazo.

Palavras-chave: Consórcio de P\&D pré-competitivo; Barreiras; Pesquisa colaborativa.

\section{Consortia and their barriers in the development of pre-competitive technologies in R\&D projects: conceptual model and application}

\begin{abstract}
This article proposes the description of the main barriers to the development of collaborative research and development (R\&D) arrangements of pre-competitive technologies. The barriers were related from the literature and interviews from members of the Friction Processing Consortium (C2PA), whose focus is on friction surfacing (FS) and friction stir welding (FSW) technologies. In addition, the degree of interference of these barriers with these cooperation models was assessed. The study results show that the barriers tend to reflect each type of formation, for example, whether they are competing or not, the maturity degree of the technologies developed, verified by the TRL (Technology Readiness Level) scale. The research revealed that the barriers considered the most harmful to consortia are those associated with lack of trust between the parties and (inadequate) management of collaboration. Likewise, there were exceptions to the difficulties arising from the innovation culture and/or production mindset in the Brazilian industry, which is not used to long-term R\&D.
\end{abstract}

Keywords: Pre-competitive R\&D consortium; Barriers; Collaborative research.

\section{Introdução}

Observa-se que as estratégias de parceria têm sido muito discutidas dentro da área de conhecimento denominada inovação aberta. Nesse contexto, Chesbrough [1] ressalta que o conhecimento pode ser encontrado em diversos

${ }^{I}$ Elo Projetos Empresariais, Belo Horizonte, MG, Brasil.

2Departamento de Pós-graduação em Engenharia Metalúrgica, Materiais e de Minas, Universidade Federal de Minas Gerais - UFMG, Belo Horizonte, $M G$, Brasil.

${ }^{3}$ Departamento de Administração, Universidade de São Paulo - USP, São Paulo, SP, Brasil.

${ }^{4}$ Embraer Commercial Aviation, São Paulo, SP, Brasil.

*Autor correspondente: reinaldolopesferreira@gmail.com

2176-1523 (C) 2021 Ferreira et al. Publicado pela ABM. Este é um artigo publicado em acesso aberto (Open Access) sob a licença Creative Commons Attribution, que permite uso, distribuição e reprodução em qualquer meio, sem restrições desde que o trabalho original seja corretamente citado. 
ambientes, seja em parcerias com clientes, fornecedores, universidades, laboratórios, consórcios, consultorias, seja até em startups. $\mathrm{O}$ autor diz ainda que "[...] as pessoas que buscam ideias novas precisam trabalhar em um cenário de receptividade em relação a todos os tipos de ideias e em uma perspectiva mais ampla com relação às necessidades da sua organização" [1].

Portanto, as alianças estratégicas se configuram como uma inovação que está transformando o foco da condução dos negócios de algumas organizações, sobretudo as que atuam globalmente, cujas relações de cooperação são amplas. Além de permitir que as empresas participantes mantenham recursos programados e compartilhem riscos, as alianças estratégicas possibilitam a criação de oportunidades para o crescimento e o incremento de competências e de poder de mercado [2].

Em consenso, Tigre [3] afirma que a formação de consórcios de $\mathrm{P} \& \mathrm{D}$ tem sido tendência mundial, devido à maior complexidade científica, à "[...] convergência tecnológica e aos altos custos das atividades de pesquisa". Por isso, as empresas estão se juntando em alianças estratégicas, com o objetivo de complementar suas competências e dividir custos e riscos, intrínsecos a todo processo de inovação. As empresas estão ficando cada vez mais envolvidas em esforços conjuntos de pesquisa, com outras indústrias e com parceiros acadêmicos e institutos de pesquisa.

Segundo Fernandez et al. [4], a principal finalidade desses modelos de cooperação, tanto públicos quanto privados, é "[...] trabalhar de forma colaborativa em projetos de escopo predefinido e de interesse comum", nas áreas de pesquisa e desenvolvimento, a fim de aprimorar a competitividade tecnológica das empresas, já que contribuem para o desenvolvimento de tecnologia de ponta. Esses autores complementam que uma das práticas em pesquisa colaborativa, é o modelo dos consórcios de P\&D pré-competitivos, formados pela junção de atividades sistemáticas, voltadas a diferentes temas de pesquisas em tecnologias.

Por sua vez, Trott [5] afirma que os consórcios de P\&D pré-competitivos contribuem para o compartilhamento dos custos e riscos de pesquisas tecnológicas que estão em estágios iniciais e sem nenhuma ideia específica do produto final, por isso o nome "pré-competitivo", ou seja, aquelas tecnologias que estão prestes a ser concluídas e incorporadas a novos produtos.

Nesse sentido, a avaliação do estágio de maturidade das tecnologias é feita pela aplicação da escala do nível de prontidão tecnológica (Technology Readiness Level - TRL), que é " [...] um sistema de medição sistemática que dá suporte à avaliação da maturidade de uma determinada tecnologia e a comparação consistente de maturidade entre diferentes tipos de tecnologia" [6]. A TRL mostra um panorama momentâneo da prontidão ou maturidade da tecnologia em estudo, além do seu grau de avanço até ser incorporada a um produto industrial.

A literatura e a pesquisa, nas quais se baseou este artigo, revelam que existem dificuldades - que podem se tornar barreiras -, principalmente na gestão desses arranjos colaborativos. As barreiras podem estar relacionadas ao gerenciamento das questões relativas às pesquisas, por exemplo, geração e incremento de ideias, transferência de conhecimento, propriedade intelectual, linhas de fomento, bem como as decisões estratégicas, por parte das empresas, sobre a escolha das tecnologias que se encontram em estágios iniciais e que poderão vir a ser transformadas em um ativo estratégico de valor [7-10].

Diante das poucas contribuições na literatura sobre arranjos de pesquisa colaborativa, especialmente os associados ao modelo de consórcio de $\mathrm{P} \& \mathrm{D}$ pré-competitivo, no ambiente industrial brasileiro, a finalidade deste artigo é identificar as principais barreiras que podem impedir o atingimento dos objetivos de consórcios de P\&D pré-competitivos.

Seguem a conceituação teórica das barreiras e a metodologia utilizada, acompanhadas da análise dos resultados das entrevistas e da avaliação das barreiras, bem como a discussão comparativa com outros autores. Por fim, as conclusões.

\subsection{Modelo conceitual}

A partir da revisão da literatura, serão expostos aspectos e situações próprios de ambientes de cooperação em P\&D pré-competitivo. São aspectos do processo de formação e desenvolvimento de arranjos colaborativos que, se não gerenciados adequadamente, podem se tornar barreiras para o sucesso dos consórcios. A Tabela 1 resume a conceituação das barreiras a consórcios de P\&D, sob o ponto de vista de diferentes autores.

\section{Metodologia}

O objeto deste estudo foi o Consórcio de Pesquisa da Tecnologia de Processamento por Atrito e Mistura (C2PA), denominado Convênio de Cooperação Científica e Tecnológica, constituído em 2015 [19]. O consórcio foi composto pelos membros industriais Empresa Brasileira de Aeronáutica (EMBRAER) e Companhia Brasileira de Alumínio (CBA); e os membros acadêmicos, Institutos de Ciência e Tecnologia (ICTs): Instituto de Pesquisas Tecnológicas do Estado de São Paulo (IPT), Centro Nacional de Pesquisas em Energias e Materiais (CNPEM) por meio do Laboratório Nacional de Nanotecnologia (LNNano); Universidade Estadual de Ponta Grossa (UEPG); a Faculdade de Ciências Aplicadas (FCA) [19], além de uma entidade de fomento, representada pela Empresa Brasileira de Pesquisa e Inovação Industrial (EMBRAPII).

A coleta dos dados foi realizada em duas fases: a primeira compreendeu a pesquisa conceitual (revisão da literatura) relacionada ao tema do artigo, e a segunda referiu-se à coleta de dados propriamente dita por meio de entrevistas em profundidade. Os entrevistados discorreram sobre sua 
Tabela 1. Barreiras de P\&D em ambiente cooperativo: modelo conceitual

\begin{tabular}{|c|c|c|}
\hline Barreiras & Síntese & Referências \\
\hline & $\begin{array}{l}\text { * Diferenças de cultura e de tecnologias costumam frustrar o sentido de unidade e acabam } \\
\text { inibindo o livre fluxo de ideias. }\end{array}$ & Smilor et al. [7] \\
\hline \multirow[t]{3}{*}{ Cultural } & $\begin{array}{l}\text { * Diferenças culturais, advindas de colaborações com parceiros de países diferentes podem } \\
\text { criar barreiras culturais, institucionais e sociais, dificultando o processo de colaboração. }\end{array}$ & Chiesa e Manzini [8] \\
\hline & $\begin{array}{l}\text { * Particularidades de cada entidade envolvida podem causar divergências que dificultam o } \\
\text { estabelecimento de objetivos comuns. }\end{array}$ & Benedetti e Torkomian [9] \\
\hline & $\begin{array}{l}\text { * Comunicação é crítica e é prejudicada quando as empresas participantes do consórcio não } \\
\text { têm capacidade para assimilar e aplicar as informações para fins comerciais. }\end{array}$ & Powell e Grodal [11] \\
\hline \multirow[t]{5}{*}{$\begin{array}{l}\text { Operacional / } \\
\text { estrutural }\end{array}$} & $\begin{array}{l}\text { * Velocidade da inovação pode sofrer variações em formações de cooperação entre empresas } \\
\text { e universidades, já que empresas estão voltadas para as necessidades e exigências dos } \\
\text { mercados, enquanto as universidades se dedicam, exclusivamente à pesquisa científica, } \\
\text { sem preocupação com aplicações imediatas. }\end{array}$ & Benedetti e Torkomian [9] \\
\hline & $\begin{array}{l}\text { * Falta de confiança, de comprometimento, liderança, transparência, comunicação e } \\
\text { monitoramento, assim como a dificuldade para identificação e gerenciamento adequado } \\
\text { das incertezas e contradições, podem comprometer o sucesso da colaboração. }\end{array}$ & Brocke e Lippe [12] \\
\hline & $\begin{array}{l}\text { * O gerenciamento inadequado do "movimento desordenado e caótico de ideias" do processo } \\
\text { de P\&D pode prejudicar o sucesso do consórcio. }\end{array}$ & Smilor et al. [7] \\
\hline & $\begin{array}{l}\text { * A falta de confiança entre as partes, de proximidade geográfica e de políticas favoráveis } \\
\text { pode dificultar a transferência de tecnologia e o aprendizado dos envolvidos. }\end{array}$ & $\begin{array}{l}\text { Santoro e Gopalakrishnan } \\
\text { [13] }\end{array}$ \\
\hline & $\begin{array}{l}\text { * Processos complexos de financiamento de } \mathrm{P} \& \mathrm{D} \text {, especialmente os dependentes de recursos } \\
\text { públicos, dificultam a implementação de projetos de } \mathrm{P} \& \mathrm{D} \text { e inovação (Brasil). }\end{array}$ & Parolin et al. [14] \\
\hline Financeira & $\begin{array}{l}\text { * Alto grau de risco e incertezas associadas a projetos de tecnologia/inovação são características } \\
\text { críticas que podem dificultar investimentos. }\end{array}$ & Ghapanchi et al. [15] \\
\hline \multirow[t]{5}{*}{$\begin{array}{c}\text { Perfil dos } \\
\text { participantes }\end{array}$} & $\begin{array}{l}\text { * Por serem formadas por "atores heterogêneos", as redes de P\&D são carregadas de } \\
\text { tensão, o que pode afetar a disposição e capacidade dos envolvidos na busca e integração } \\
\text { de conhecimento. }\end{array}$ & $\begin{array}{l}\text { Corsaro et al. [16]; } \\
\text { Ritala et al. [10]; Chiesa e } \\
\text { Manzini [8] }\end{array}$ \\
\hline & $\begin{array}{l}\text { * Ambiente com maior nível de competição provoca redução efetiva do nível de incremento } \\
\text { de P\&D. }\end{array}$ & $\begin{array}{l}\text { Branstetter e Sakakibara } \\
\qquad[17]\end{array}$ \\
\hline & $\begin{array}{l}\text { * Arranjos no formato empresa-empresa podem ser menos produtivos do que entre empresas } \\
\text { e universidades. }\end{array}$ & Santoro [18] \\
\hline & $\begin{array}{l}\text { * Estrutura organizacional e objetivos da pesquisa do consórcio são barreiras à transferência } \\
\text { de tecnologia. }\end{array}$ & Smilor et al. [7] \\
\hline & $\begin{array}{l}\text { * Discordâncias acerca do objetivo e conteúdo da colaboração podem comprometer o } \\
\text { sucesso do consórcio. }\end{array}$ & Chiesa e Manzini [8] \\
\hline \multirow[t]{2}{*}{ Estratégica } & $\begin{array}{l}\text { * O nível de controle (proteção) exigido pelas tecnologias desenvolvidas, implica na decisão } \\
\text { das empresas de manter acesso restrito (interno) ou contratar parceiros externos. }\end{array}$ & Trott [5] \\
\hline & $\begin{array}{l}\text { * Tipo de conhecimento transferido e adquirido, bem como o nível de inovação alcançado } \\
\text { (nova ou incremental), interferem no custo da transferência de conhecimento. }\end{array}$ & Ritala et al. [10] \\
\hline
\end{tabular}

experiência prática em consórcios e seu conhecimento geral a respeito do assunto e comentaram as eventuais barreiras.

As barreiras identificadas para o estudo conceitual foram juntadas às barreiras citadas pelos entrevistados e, mediante um instrumento desenvolvido pelos autores do artigo, foi solicitada a avaliação do grau de interferência dessas barreiras no desenvolvimento e no sucesso de um consórcio de P\&D pré-competitivo. Utilizou-se uma escala de 5 (cinco) pontos, cujas extremidades indicam que determinada barreira pode gerar muito (5) ou nenhum (1) grau de interferência ao desenvolvimento do consórcio. Além de avaliar o grau de interferência das barreiras, os entrevistados comentaram suas respostas.

As entrevistas aconteceram em períodos aleatórios. Entre o primeiro semestre de 2016 e o início do segundo semestre de 2019, foram orientadas por um roteiro de abordagens baseadas no modelo conceitual e de acordo com os objetivos do artigo. O tratamento dos dados foi realizado de forma a estruturar e analisar as informações obtidas e balizá-las frente às fontes conceituais [20].

\section{Resultados}

\subsection{Contexto}

Algumas das barreiras identificadas no C2PA coincidiram com as referidas pela literatura e outras são específicas do C2PA, seja por causa das características da sua configuração, seja por causa do cenário da inovação no Brasil.

Tratando-se de pesquisa em fases pré-competitivas, as dificuldades podem ser muitas porque seu o custo é elevado, e os resultados são obtidos após longo período de desenvolvimento, em oposição ao ritmo e às expectativas, 
sobretudo da indústria, que se empenha para atender demandas urgentes do mercado.

O consórcio sofreu com algumas dificuldades, mas foram inferiores em comparação com os resultados positivos obtidos - suficientes para que os entrevistados ressaltassem a importância do consórcio para as empresas participantes e para o progresso de projetos de $\mathrm{P} \& \mathrm{D}$ pré-competitivos no Brasil. Cada empresa associada tem sua perspectiva própria, mas elas concordam que a reunião do conhecimento acadêmico com o "ferramental" dos Institutos de Ciência e Tecnologia (ICTs), e o drive que a indústria proporciona, no sentido de direcionar as pesquisas para atender as demandas do mercado, "é a receita para o sucesso" na busca de competitividade. A opinião comum entre os entrevistados é que esse modelo de colaboração já está provocando "mudança de mindset" e instigará as empresas a "[...] pensar em pesquisa aplicada a seu portfólio, mas não com vistas em curtíssimo prazo". Ademais, o consórcio pré-competitivo produz novas tecnologias, e a "geração de tecnologias é o que mantém as empresas na vanguarda".

\subsection{Interferência das barreiras ao desenvolvimento e sucesso de consórcios de $P \& D$}

A Tabela 2 apresenta a lista das barreiras mais discutidas pelos autores constantes do modelo conceitual e as citadas pelos entrevistados. Com relação a algumas das barreiras listadas, a avaliação foi feita para um contexto genérico e não somente baseada no C2PA, já que o consórcio pesquisado apresentou poucas dificuldades até o período em análise - apenas as barreiras 9 e 10 foram mencionadas espontaneamente pelos entrevistados. As demais são originadas do referencial teórico.
De modo geral, são consensuais as opiniões sobre as barreiras, embora tenha sido verificada alguma diferença de percepção de determinadas barreiras voltadas para a atividade principal dos públicos entrevistados. Por exemplo, percebeu-se que as universidades e os institutos de pesquisa são mais sensíveis a questões de atrasos e cumprimento de tarefas/etapas, pois estão diretamente envolvidos com o prazo dos "entregáveis" das pesquisas desenvolvidas. Por outro lado, os representantes da indústria manifestaram mais preocupação com ambientes (de pesquisa) em que haja falta de confiança entre as partes, sobretudo no que se refere a compartilhamento de conhecimento com membros participantes concorrentes.

Nesse sentido, foi destacada a importância da governança do consórcio para o seu desenvolvimento e sucesso, assim como a capacidade de liderança da pessoa responsável por essa função, que tem o papel de agregar o grupo e dirimir conflitos que surjam durante o processo de desenvolvimento do consórcio. Dessa maneira, questões culturais, de confiança, de liderança e de heterogeneidade dos participantes do consórcio terão importância maior ou menor e poderão comprometer ou não a colaboração, conforme o plano e a aplicação das ações de governança previstas. $\mathrm{O}$ que pode ser constatado pela grande relevância dada às barreiras 3 e 2 (grau de interferência 5 e 4, respectivamente).

Das barreiras avaliadas, 5 foram consideradas de média relevância (grau de interferência 3) para o desenvolvimento do consórcio, enquanto 3 barreiras foram vistas com relevância média-alta (grau de interferência 4) e, por fim, uma barreira, de alta relevância (grau de interferência 5).

A percepção dos entrevistados sobre cada barreira da Tabela 2 é a seguinte:

Tabela 2. Grau de interferência no desenvolvimento e sucesso de um consórcio de P\&D

\begin{tabular}{|c|c|c|}
\hline & Barreiras & $\begin{array}{c}\text { Grau de } \\
\text { interferência }\end{array}$ \\
\hline 1 & $\begin{array}{l}\text { As diferenças culturais, organizacionais ou de particularidades de cada entidade envolvida podem dificultar a } \\
\text { colaboraça. }\end{array}$ & 3 \\
\hline 2 & $\begin{array}{l}\text { O gerenciamento inadequado da troca de ideias e conhecimento pode prejudicar o desenvolvimento e o sucesso } \\
\text { do consórcio. }\end{array}$ & 4 \\
\hline 3 & $\begin{array}{l}\text { A falta de confiança, de comprometimento, liderança, transparência, comunicação e monitoramento pode comprometer } \\
\text { o sucesso da colaboração. }\end{array}$ & 5 \\
\hline 4 & $\begin{array}{l}\text { O alto grau de risco e as incertezas associadas a projetos de tecnologia/inovação são características críticas que } \\
\text { podem dificultar investimentos. }\end{array}$ & 3 \\
\hline 5 & $\begin{array}{l}\text { Por serem formados por "atores heterogêneos" os consórcios de P\&D são carregados de tensão, o que pode afetar } \\
\text { a disposição e a capacidade dos envolvidos em busca da integração de conhecimento. }\end{array}$ & 3 \\
\hline 6 & A estrutura organizacional e os objetivos da pesquisa do consórcio são barreiras à transferência de tecnologia. & 3 \\
\hline 7 & $\begin{array}{l}\text { O nível de controle (proteção) exigido pelas tecnologias desenvolvidas interfere na decisão das empresas de manter } \\
\text { acesso restrito (interno) ou contratar parceiros externos. }\end{array}$ & 2 \\
\hline 8 & $\begin{array}{l}\text { O tipo de conhecimento transferido e adquirido, bem como o nível de inovação alcançado (nova ou incremental) } \\
\text { interferem no custo da transferência de conhecimento. }\end{array}$ & 3 \\
\hline 9 & $\begin{array}{l}\text { O atraso em alguma etapa durante o desenvolvimento do consórcio pode comprometer os prazos dos processos } \\
\text { seguintes. }\end{array}$ & 4 \\
\hline 10 & $\begin{array}{l}\text { A dificuldade para mudança de mindset com relação ao mercado e os riscos inerentes ao desenvolvimento de } \\
\text { tecnologias pré-competitivas podem induzir as empresas a manter a cultura de produção de commodities em vez } \\
\text { de criar mercados para novas tecnologias. }\end{array}$ & 4 \\
\hline
\end{tabular}


Barreira 1: A princípio, é vista como uma barreira que deve ser considerada com atenção, porém a maioria dos entrevistados acha que as diferenças culturais entre os membros do consórcio podem ser resolvidas à medida que aumentam o contato e o convívio entre os envolvidos, por isso a média baixa de interferência no desenvolvimento e no sucesso do consórcio. É uma barreira que pode dificultar o desenvolvimento do consórcio, mas não inviabilizá-lo. Há quem afirme que essas diferenças estão associadas à gestão, portanto podem ser evitadas se o consórcio for "bem coordenado" (gerido). Nesse sentido, Benedetti e Torkomian [9] previram que as especificidades de cada membro participante de consórcios podem causar divergências que prejudiquem a definição de objetivos comuns;

Barreira 2: A troca de ideias e conhecimento é vista como natural e importante no ambiente de consórcio, "é um motivador para a colaboração". É consenso que, assim como as diferenças culturais, o "alinhamento" de objetivos e ideias são alcançados e aprimorados com o tempo de convívio. O importante é perceber que, se não houver gerenciamento adequado das ideias - que costumam estar desordenadas -, o sucesso do consórcio pode ser comprometido [7];

Barreira 3: Para os entrevistados, "se não houver confiança e comprometimento, o projeto não anda", e isso depende muito de "uma boa liderança" para conduzir os trabalhos. A liderança é considerada um quesito importante e está fundamentalmente associada à confiança (entre as partes) para a troca de informações e os conhecimentos, além do comprometimento de cada participante com seu papel e sua responsabilidade no consórcio. Essa percepção é corroborada por Brocke e Lippe [12], que acreditam que a falta de confiança, de comprometimento, liderança, transparência, comunicação e monitoramento [...] pode dificultar o sucesso da colaboração;

Barreira 4: Se o risco é alto, a tendência é haver dificuldade para investimentos e participação de empresas. Especialmente no Brasil, em que as empresas "preferem trabalhar com algo mais seguro", sem riscos ou incertezas, daí a cultura de produção de commodities. O alto grau de risco e incertezas inerentes a esses tipos de pesquisas acabam se tornando uma característica crítica para a tomada de decisão dos investidores [15];

Barreira 5: Na maior parte dos casos, esta barreira é vista como saudável, já que a opinião geral é que as diferenças acabam sendo desfeitas ou se transformam em aprendizado - por exemplo, contribuem para um olhar mais atento (não percebido antes) a situações, processos e até a tecnologias desenvolvidas (que ainda não haviam despertado interesse). Houve quem tivesse chamado atenção para o fato de que a tensão por causa da heterogeneidade dos atores vai interferir (ou não) de forma diferente, conforme cada tipo ou formação de consórcio, ou seja, depende do grau de alinhamento entre os participantes e dos objetivos traçados. Sendo assim, alguns autores ressaltam que a tensão provocada pelas diferenças entre os participantes do consórcio pode afetar a disposição e a capacidade dos envolvidos na busca e integração de conhecimento $[8,10,16]$;

Barreira 6: Três questões importantes foram destacadas como preocupação para esta barreira: (1) a estrutura organizacional do consórcio deve "ser condizente com os objetivos" propostos, no sentido de ser suficiente para suportar as necessidades das pesquisas; (2) a estrutura do consórcio deve ser proporcional ao "tamanho do grupo" participante, a fim de facilitar a participação igualitária de todos os membros, principalmente nas tomadas de decisão; (3) a estrutura do consórcio e os objetivos da pesquisa devem estar bem definidos ("até onde se pode ir"), de modo que os interesses de alguns associados não se sobreponham aos dos outros - "é preciso esclarecer os interesses", para evitar conflitos. Nesse caso, a estrutura organizacional e os objetivos da pesquisa desenvolvida pelo consórcio podem ser barreiras à transferência de tecnologia entre os participantes [7]. Da mesma forma, as discordâncias sobre os objetivos e o conteúdo da colaboração também podem dificultar o bom desenvolvimento do consórcio [8];

Barreira 7: Em projetos com desenvolvimento em fases pré-competitivas (como é o caso do C2PA), não há preocupação com sigilo, até porque as tecnologias não estão claramente definidas - daí, a baixa relevância dada a essa barreira (grau de interferência 2). A busca por registro de propriedade intelectual é maior nas pesquisas em fases avançadas de maturidade, quando as tecnologias estão próximas a se transformar em produto. Nesses casos, as empresas naturalmente internalizam suas pesquisas. Portanto, conforme o nível de proteção exigido pelas tecnologias desenvolvidas, as empresas devem decidir se mantêm acesso restrito (interno) ou contratam parceiros externos [5];

Barreira 8: Assim como a tecnologia desenvolvida, o conhecimento compartilhado durante o processo de desenvolvimento, e a expertise de cada membro do consórcio, podem interferir no custo do conhecimento. Além disso, há fatores que aumentam o custo do conhecimento gerado e transferido: se a tecnologia é complexa ("disruptiva"), se exige "olhar multidisciplinar" ou se o desenvolvimento está em fases pré-competitivas. Ritala et al. [10] afirmam que o tipo de conhecimento transferido e adquirido, bem como o nível de inovação alcançado (nova ou 
incremental), são fatores que interferem no custo da transferência de conhecimento;

Barreira 9: Os prazos de cronograma de P\&D são vistos sob pontos de vista relativos, a depender de circunstâncias. Há consenso de que a pesquisa de longo prazo demanda muitas fases, testes e experimentações, portanto não se pode exigir ou esperar rapidez, pois nesse tipo de consórcio "a rota, a direção, é mais importante que a velocidade". Portanto, atrasos em P\&D são 'normais' e geralmente "as partes se comunicam, se justificam e pensam juntas em como dar o próximo passo ou contornar a dificuldade". Todavia, a percepção é diferente para alguns membros de universidades e institutos de pesquisa, que dão mais importância ao cumprimento de prazos, já que eles são responsáveis diretos pelo desenvolvimento das pesquisas. Sem desconsiderar a relevância dos processos de longo prazo, inerentes ao arranjo $\mathrm{P} \& \mathrm{D}$ pré-competitivo, eles chamam atenção para a questão específica de atrasos em atividades de trabalho que compõem a "cadeia" de P\&D, estas sim, se atrasadas, acabam comprometendo etapas seguintes dos processos e a própria "agenda" do consórcio;

Barreira 10: A opinião comum é que a mentalidade da indústria brasileira ainda tem que evoluir bastante, para sair da expectativa de retorno financeiro imediato, produzindo commodities, e passar a desenvolver e criar produtos inéditos e inovadores (que agreguem valor ao seu negócio). Assim como em outras barreiras, foi ressaltado que essa situação não se aplica a pesquisas pré-competitivas, já que “[...] as empresas só deveriam pensar efetivamente no mercado se (quando) estivessem preocupadas em desenvolver produto". Em colaborações entre empresas e universidades, a velocidade da inovação pode variar, porque, segundo Benedetti e Torkomian [9], o interesse das empresas está direcionado para as necessidades e as exigências dos mercados, enquanto as universidades estão voltadas para a pesquisa científica, sem preocupação com aplicação imediata [9].

\section{Considerações finais}

Embora seja incipiente no Brasil, o modelo de consórcios de $\mathrm{P} \& \mathrm{D}$ pré-competitivos tem proporcionado o desenvolvimento da pesquisa de longo prazo e em fases iniciais de desenvolvimento, o que adiciona componente de inovação ao trabalho dos pesquisadores e, sobretudo, das empresas envolvidas. Pelo menos entre os atores que estão engajados no ambiente de Pesquisa, Desenvolvimento e Inovação (PD\&I), esse é um movimento que provoca otimismo com relação ao que está por vir.
Baseadas nos resultados da pesquisa realizada, as principais barreiras ao desenvolvimento de um consórcio de $P \& D$ pré-competitivo antecedem o processo de pesquisa e desenvolvimento de novas tecnologias, a saber:

i) No Brasil, antes de enfrentar as barreiras inerentes ao ambiente interno dos consórcios e aos processos para sua operacionalização, os interessados na formação desse tipo de arranjo colaborativo precisam vencer os obstáculos decorrentes das políticas de incentivo às pesquisas de inovação e, sobretudo, da falta de cultura e de compreensão dos governos e de empresas acerca da importância da pesquisa de longo prazo para o desenvolvimento e a competitividade do País e da indústria nacional. A indústria brasileira ainda concentra sua produção em commodities. Contrariamente ao propósito de consórcios de P\&D pré-competitivos ("olhar para o futuro"), a postura de inovação da maioria das empresas brasileiras tende a estar baseada nas pressões do mercado e nas demandas - urgentes - requeridas por seus clientes. Daí a necessidade de mudança de mindset das empresas. O Brasil precisa deixar de "sempre copiar o outro" e começar a "ser vanguarda". É a inovação que propicia essa transformação. Evoluir da produção de commodities para a criação de produtos e serviços inovativos, que inclusive poderão exigir a criação de novos mercados, específicos para tecnologias promissoras, que sejam descobertas ou desenvolvidas nos ambientes de arranjos colaborativos;

ii) Observa-se que as universidades carecem de uma visão de negócio (que a priori não é sua atribuição), pois essa condição tem se tornado de extrema relevância, especialmente pelas constantes instabilidades e incertezas na área de pesquisa científica brasileira, o que sempre exigiu 'jogo de cintura' e capacidade dessas instituições de ensino para buscar e negociar recursos para seus projetos de pesquisa. Do lado da indústria, o que se percebe é que, além de 'aprender' sobre consórcios, as empresas brasileiras precisarão incorporar, efetivamente, uma cultura de "paciência" e planejamento aos seus processos de PD\&I. Ao decidir participar de um consórcio de $\mathrm{P} \& \mathrm{D}$ pré-competitivo, é importante que as empresas já tenham assimilado a ideia de que as respostas para seus objetivos não serão imediatas. Assim como empresas, ICTs e universidades deveriam priorizar o trabalho conjunto, no sentido de fazer com que a evolução do conhecimento sobre um determinado problema e a aquisição de conhecimentos científicos associados a um problema real, conduzam à produção de inovação.

Não há dúvidas sobre as dificuldades inerentes a esse tipo de cooperação e, sabe-se, o caminho é longo, mas as iniciativas já implementadas e em andamento provocam 
otimismo e fazem acreditar que é possível realizar projetos de inovação no Brasil.

Os autores deste artigo agradecem aos membros do Consórcio de Pesquisa da Tecnologia de Processamento por Atrito e Mistura (C2PA), que disponibilizaram parte do seu tempo para ceder informações relevantes para esta pesquisa, e às instituições CAPES, PROEX, CNPq e FAPEMIG.

\section{Referências}

1 Chesbrough H. Inovação aberta: como criar e lucrar com a tecnologia. Porto Alegre: Bookman; 2012.

2 Hagedoorn J, Schakenraad J. Interfirm partnerships for generic technologies: the case of new materials. Technovation. 1991;11(7):429-444.

3 Tigre PB. Gestão da inovação: a economia da tecnologia no Brasil. Rio de Janeiro: Campus; 2006.

4 Fernandez FF, Vasconcellos E, Guedes LF, Carlana R, Matta V. Long-term R\&D-based consortia: paths to integrate basic research with company strategy. In: Proceedings of the of the $25^{\text {th }}$ International Conference for Management of Technology; 2016; Orlando, FL. Orlando: IAMOT; 2016. p. 973-992.

5 Trott P. Gestão da inovação e desenvolvimento de novos produtos. 4. ed. Porto Alegre: Bookman; 2012.

6 Mankins JC. Technology readiness levels: a white paper. Washington, DC: NASA; 1995 [acesso em 3 jan. 2013]. Disponível em: http://www.hq.nasa.gov/office/codeq/trl/trl.pdf

7 Smilor RW, Gibson DV, Avery CMR. R\&D consortia and technology transfer: initial lessons from MCC. The Journal of Technology Transfer. 1989;14(2):11-22.

8 Chiesa V, Manzini R. Organizing for technological collaborations: a managerial perspective. R\&D Management. 1998;28(3):199-212.

9 Benedetti MH, Torkomian ALV. Uma análise da influência da cooperação universidade-empresa sobre a inovação tecnológica. Gestão \& Produção. 2010;17(4):1-14.

10 Ritala P, Huizingh E, Wijbenga P. Participating R\&D consortia with without competitors. In: Proceedings of the ISPIM Asia-Pacific Innovation Forum; 2014; Manchester. Manchester: ISPIM; 2014.

11 Powell WW, Grodal S. Networks of innovators. In: Fagerberg J, Mowery DC, Nelson RR, editors. The Oxford handbook of innovation. New York: Oxford University Press; 2005. cap. 3, p. 56-85.

12 Brocke J, Lippe S. Managing collaborative research projects: a synthesis of project management literature and directives for future research. International Journal of Project Management. 2015;33(5):1022-1039.

13 Santoro MD, Gopalakrishnan S. Relationship dynamics between university research centers and industrial firms: their impact on technology transfer activities. The Journal of Technology Transfer. 2001;26(1-2):163-171.

14 Parolin SRH, Vasconcellos E, Bordignon JA. Barreiras e facilitadores à inovação: o caso Nutrimental S. A. Revista de Economia Mackenzie. 2006;4(4):12-34.

15 Ghapanchi AH, Tavana M, Khakbaz MH, Low G. A methodology for selecting portfolios of projects with interactions and under uncertainty. International Journal of Project Management. 2012;30(7):791-803.

16 Corsaro D, Cantú C, Tunisini A. Actors' heterogeneity in innovation networks. Industrial Marketing Management. 2012;41(5):780-789.

17 Branstetter LG, Sakakibara M. When do research consortia work well and why? Evidence from Japanese panel data. The American Economic Review. 2002;92(1):143-159.

18 Santoro MD. Success breeds success: the linkage between relationship intensity and tangible outcomes in industryuniversity collaborative ventures. The Journal of High Technology Management Research. 2000;11(2):255-273.

19 Convênio de Cooperação Científica e Tecnológica. VDT055-12. Campinas; 2015. 20 p. (Mimeo).

20 Vergara SC. Projetos e relatórios de pesquisa em administração. 3.ed. São Paulo: Atlas; 2000.

Recebido em: 22 Jun. 2020

Aceito em: 13 Set. 2020 